$$
\begin{aligned}
& \text { MCS-P-451-0794 } \\
& \text { \&Ob y } \\
& \text { Nov } 05 \text { iss } \\
& \text { OSTI }
\end{aligned}
$$

\title{
STENMIN: A Software Package for Large, Sparse Unconstrained Optimization Using Tensor Methods *
}

\author{
Ali Bouaricha ${ }^{\dagger}$
}

\author{
Argonne National Laboratory
}

We describe a new package for minimizing an unconstrained nonlinear function where the Hessian is large and sparse. The software allows the user to select between a tensor method and a standard method based upon a quadratic model. The tensor method models the objective function by a fourth-order model, where the third-and fourth-order terms are chosen such that the extra cost of forming and solving the model is small. The new contribution of this package consists of the incorporation of an entirely new way of minimizing the tensor model that makes it suitable for solving large, sparse optimization problems efficiently. The test results indicate that, in general. the tensor method is significantly more efficient and more reliable than the standard Newton method for solving large. sparse unconstrained optimization problems.

Categories and Subject Descriptors: G.1.3 [Numerical Analysis]: Numerical Linear Algebrasparse and very large systems; G.1.6 [Numerical Analysis]: Optimization-unconstrained optimization; (i.t [Mathematics of Computing]: Mathematical Software

Cieneral Terms: Algorithms

Ailditional hey Words and Phrases: tensor methods. sparse problems, large-scale optimization. rank-deticient matrices

\footnotetext{
- Part of this work was performed while the author was research associate at CERFACS (Centre Européen de Recherche et de Formation Avancée en (Calcul Scientifique, Toulouse, France).

${ }^{\dagger}$ Author's address: Mathematics and Computer Science Division, Argonne National Laboratory, Argonne, Illinois, 60439. bouarich 19 mcs.anl.gov. This work was supported in part by the Office of Scientific Computing. U.S. Department of Energy, under Contract W-31-109-Eng-38.
} 


\section{DISCLAIMER}

This report was prepared as an account of work sponsored by an agency of the United States Government. Neither the United States Government nor any agency thereof, nor any of their employees, makes any warranty, express or implied, or assumes any legal liability or responsibility for the accuracy, completeness, or usefulness of any information, apparatus, product, or process disclosed, or represents that its use would not infringe privately owned rights. Reference herein to any specific commercial product, process, or service by trade name, trademark, manufacturer, or otherwise does not necessarily constitute or imply its endorsement, recommendation, or favoring by the United States Government or any agency thereof. The views and opinions of authors expressed herein do not necessarily state or reflect those of the United States Government or any agency thereof. 


\section{DISCLAIMER}

Portions of this document may be illegible in electronic image products. Images are produced from the best available original document. 


\section{Introduction}

This paper describes a software package for solving the unconstrained optimization problem

$$
\text { given } f: \Re^{n} \rightarrow \Re \text {, find } x_{*} \in \Re^{n} \text { such that } f\left(x_{*}\right) \leq f(x) \text { for all } x \in D,
$$

using tensor methods, where $D$ is some open set containing $x_{*}$. We assume that $f$ is at least twice continuously differentiable and $\nabla^{2} f\left(x_{c}\right)$ is large and sparse.

Tensor methods for unconstrained optimization are general-purpose methods primarily intended to improve upon the performance of standard methods especially on problems where $\nabla^{2} f\left(x_{*}\right)$ has a small rank deficiency, and to be at least-as efficient as standard methods on problems where $\nabla^{2} f\left(x_{*}\right)$ is nonsingular. Tensor methods for unconstrained optimization base each iteration upon the fourth-order model of the objective function $f(x)$

$$
M_{T}\left(x_{c}+d\right)=f\left(x_{c}\right)+\nabla f\left(x_{c}\right) \cdot d+\frac{1}{2} \nabla^{2} f\left(x_{c}\right) \cdot d^{2}+\frac{1}{6} T_{c} \cdot d^{3}+\frac{1}{24} V_{c} \cdot d^{4},
$$

where $d \in \Re^{n}, x_{c}$ is the current iterate, $\nabla f\left(x_{c}\right)$ and $\nabla^{2} f\left(x_{c}\right)$ are the first and second analytic derivatives of $f$ at $x_{c}$, or finite difference approximations to them, and the tensor terms at $x_{c}$, $T_{c} \in \Re^{n \times n \times n}$ and $V_{c} \in \Re^{n \times n \times n \times n}$, are symmetric. (We use the notation $\nabla f\left(x_{c}\right) \cdot d$ for $\nabla f\left(x_{c}\right)^{T} d$, and $\nabla^{2} f\left(x_{c}\right) \cdot d^{2}$ for $d^{T} \nabla^{2} f\left(x_{c}\right) d$ to be consistent with the tensor notation $T_{c} \cdot d^{3}$ and $V_{c} \cdot d^{4}$. We abbreviate terms of the form $d d, d d d$, and $d d d d$ by $d^{2}, d^{3}$, and $d^{4}$, respectively.)

Schnabel and Chow [11] select $T_{c}$ and $V_{c}$ such that the model interpolates function and gradient values from $p$ past iterates, where $p$ is a small number. This strategy results in $T_{c}$ and $V_{c}$ being low-rank tensors, which is crucial for the efficiency of the tensor method. Here, we consider only the case where the tensor model interpolates $f(x)$ and $\nabla f(x)$ at the previous iterate (i.e., $p=1$ ). The reasons for this choice are that the performance of the tensor version that allows $p \geq 1$ is similar overall to that constraining $p$ to be 1 , and that the method is simpler and less expensive to implement in this case.

The above choice of $T_{c}$ and $V_{c}$ yields the tensor model

$$
M_{T}\left(x_{c}+d\right)=f\left(x_{c}\right)+\nabla f\left(x_{c}\right) \cdot d+\frac{1}{2} \nabla^{2} f\left(x_{c}\right) \cdot d^{2}+\frac{1}{2}\left(b^{T} d\right)\left(s^{T} d\right)^{2}+\frac{\gamma}{24}\left(s^{T} d\right)^{4},
$$

where $s \in \Re^{n}$ is the step from $x_{c}$ to the previous iterate $x_{-1}$ (i.e., $s=x_{-1}-x_{c}$ ) and $b \in \Re^{n}$ and $\gamma \in \mathscr{R}$ are uniquely determined by the requirements $M_{T}\left(x_{-1}\right)=f\left(x_{-1}\right)$ and $\nabla M_{T}\left(x_{-1}\right)=$ $\nabla f\left(x_{-1}\right)$. The whole process of forming the tensor model requires only $O\left(n^{2}\right)$ arithmetic operations. The storage needed for forming and storing the tensor model is only a total of $6 n$.

The tensor algorithus described in [11] are QR-based algorithms involving orthogonal transformations of the variable space. These algorithms are very effective for minimizing the tensor model when the Hessian is dense because they are very stable numerically, especially when the Hessian is singular. They are not efficient for sparse problems, however, because they destroy the sparsity of the Hessian due to the orthogonal transformation of the variable space. To preserve the sparsity of the Hessian, we developed in [4] an entirely new way of minimizing the tensor model that employs a sparse variant of the Cholesky decomposition. This makes the new algorithms very well suited for sparse problems. In this new approach, we show that the minimization of (1.3) can be reduced to the solution of a third-order polynomial in one unknown, 
plus the solution of three systems of linear equations that all involve the same coefficient matrix $\nabla^{2} f\left(x_{c}\right)$. The STENMIN package is essentially based on this new approach.

The remainder of this paper is organized as follows. In $\S 2$ an iteration of tensor methods for large, sparse unconstrained optimization is outlined. In $\$ 3$ we give an overview of the input, output, and important options provided by the software package. We describe the user interface to the package in $\S 4$, which includes both a simplified (default) and a longer calling sequence. In $\$ 5$ we describe the meaning of the input, input-output, and output parameters for the package. In $\S 6$ we present the default values provided by the package. A few implementation dependencies are described in $\S 7$. In $\S \S$ we give an example of the use of the package. Finally, in $\S 9$ we describe comparative testing for an implementation based on the tensor method versus an implementation based on the Newton's method, and we present summary statistics of the test results.

\section{An Iteration of Tensor Methods}

In this section, we present the overall algorithm for tensor methods for large, sparse unconstrained optimization. Algorithm 2.1 is a slightly modified version of the algorithm described in [4] in the way the tensor step is selected when the $\beta$ equation (see algorithm below) has more than one root. In general, this new way of computing the tensor step appears to perform better than the strategy described in [4], in both function evaluations and execution times. A summary of the experimental results for this implementation is presented in $§ 9$.

\section{Algorithm 2.1. An Iteration of Tensor Methods for Large, Sparse Unconstrained Optimization}

Let $x_{c}$ be the current iterate, $x_{+}$the next iterate, $d_{t}$ the tensor step, and $d_{n}$ the Newton step.

1. Calculate $\nabla f\left(x_{c}\right)$, and decide whether to stop. If not:

2. Calculate $\nabla^{2} f\left(x_{c}\right)$

3. Calculate $b$ and $\gamma$ in the tensor model (1.3), so that the tensor model interpolates $f(x)$ and $\nabla f(x)$ at $x_{-1}$

4. Find a potential minimizer $d_{t}$ of the tensor model

4.1. Factor $\nabla^{2} f\left(x_{c}\right)$ using the MA27 package [8]

4.2. if $\nabla^{2} f\left(x_{\varepsilon}\right)$ has full rank then

4.2.1. Form the $\beta$ equation $(\beta \in \Re)$ :

$$
-u+(y w-u v-1) \beta-\frac{3}{2} v \beta^{2}+\left(\frac{1}{2} w z-\frac{\gamma}{6} w-\frac{1}{2} v^{2}\right) \beta^{3}
$$

where $u=s^{T} \nabla^{2} f\left(x_{c}\right)^{-1} \nabla f\left(x_{c}\right), v=s^{T} \nabla^{2} f\left(x_{c}\right)^{-1} b, w=s^{T} \nabla^{2} f\left(x_{c}\right)^{-1} s$, $y=b^{T} \nabla^{2} f\left(x_{c}\right)^{-1} \nabla f\left(x_{c}\right)$, and $z=b^{T} \nabla^{2} f\left(x_{c}\right)^{-1} b$

4.2.2. (Compute the roots of the $\beta$ equation

4.2.3. Select $\beta_{*}=\min \left(\left|\beta_{i}\right|\right)$ where $\beta_{i}$ are the roots of the $\beta$ equation

4.2.4. Substitute $\beta_{*}$ into

$$
\theta_{*}=-\frac{\left(u+\beta_{*}+\frac{1}{2} v \beta_{*}^{2}+\frac{\gamma}{6} w / \beta_{*}^{3}\right)}{w / \beta_{*}}
$$

4.2.5. Calculate the tensor step:

$$
d_{t}=-\nabla^{2} f\left(x_{c}\right)^{-1}\left(\nabla f\left(x_{c}\right)+\theta_{*} \beta_{*} s+\frac{1}{2} \beta_{*}^{2} b+\frac{\gamma}{6} \beta_{*}^{3} s\right)
$$

4.3. elseif $\nabla^{2} f\left(x_{c}\right)$ is singular with $\operatorname{rank}\left(\nabla^{2} f\left(x_{c}\right)\right)=n-1$ then

4.3.1. Form the $\beta$ equation $(\beta \in \Re)$ : 


$$
u+(1+\hat{\beta} v) \beta+\left(\frac{1}{2} v+\frac{\gamma}{2} w \hat{\beta}\right) \beta^{2}+\frac{\gamma}{6} w \beta^{3}
$$

where $u=s^{T} \hat{\nabla}^{2} f\left(x_{c}\right)^{-1} \frac{\nabla}{\nabla} f\left(x_{c}\right), \hat{\nabla}^{2} f\left(x_{c}\right)=\nabla^{2} f\left(x_{c}\right)+s s^{T}$,

$\hat{\nabla} f\left(x_{c}\right)=\nabla f\left(x_{c}\right)+\nabla^{2} f\left(x_{c}\right) \hat{d}+\hat{\theta} \hat{\beta} s+\frac{1}{2} \hat{\beta}^{2} b+\frac{\gamma}{6} \hat{\beta}^{3} s, \hat{\beta}=s^{T} \hat{d}, \hat{\theta}=b^{T} \hat{d}, \hat{d}$ is the global step computed in the previous iteration, $v=s^{T} \hat{\nabla}^{2} f\left(x_{c}\right)^{-1} b$, and $w=s^{T} \hat{\nabla}^{2} f\left(x_{c}\right)^{-1} s$

4.3.2. Compute the roots of the $\beta$ equation

4.3.3. Select $\beta_{*}=\min \left(\left|\beta_{i}\right|\right)$ where $\beta_{i}$ are the roots of the $\beta$ equation

4.3.4. Substitute $\beta_{*}$ into

$$
\begin{aligned}
& \qquad \theta_{*}=\frac{1}{w\left(\hat{\beta}+\beta_{*}\right)}\left(y w \hat{\beta}-u-u v \hat{\beta}+\left(y w+z w \hat{\beta}^{2}-2 v \hat{\beta}-v^{2} \hat{\beta}^{2}-u v-1\right) \beta_{*}\right. \\
& \left.\left.+\left(\frac{3}{2} z w \hat{\beta}-\frac{\gamma}{2} w \hat{\beta}-\frac{3}{2} v-\frac{3}{2} v^{2} \hat{\beta}\right)+\frac{1}{2} z w-\frac{\gamma}{6} w-\frac{v^{2}}{2}\right) \beta_{*}^{3}\right), \\
& \text { where } y=b^{T} \hat{\nabla}^{2} f\left(x_{c}\right)^{-1} \hat{\nabla} f\left(x_{c}\right) \text {, and } z=b^{T} \hat{\nabla}^{2} f\left(x_{c}\right)^{-1} b
\end{aligned}
$$

4.3.5. Calculate the tensor step of the transformed tensor model (2.1) below

$$
\delta=-\hat{\nabla}^{2} f\left(x_{c}\right)^{-1}\left(\hat{\nabla} f\left(x_{c}\right)+\hat{\beta} \beta_{*} b+\hat{\beta} \theta_{*} s+\beta_{*} \theta_{*} s+\left(\frac{1}{2} b+\frac{\gamma}{2} \hat{\beta} s\right) \beta_{*}^{2}+\frac{\gamma}{6} \beta_{*}^{3} s\right)
$$

4.3.6. Calculate the tensor step of the original model (1.3) :

$$
d_{t}=\delta+\hat{d}
$$

4.4. else $\left\{\operatorname{rank}\left(\nabla^{2} f\left(x_{c}\right)\right)<n-1\right\}$

4.4.1. Modify the negative eigencomponents of $\nabla^{2} f\left(x_{c}\right)$

4.4.2. Perform steps $4.2 .1-4.2 .5$ endif

5. Compute a next iterate $x_{+}$

5.1. if $d_{t}$ is descent then

$x_{+}^{t}=x_{c}+d_{t}$

if $f\left(x_{+}^{t}\right)<f\left(x_{c}\right)+10^{-4} \cdot \nabla f\left(x_{c}\right)^{T} d_{t}$ then

$$
x_{+}=x_{+}^{t}
$$

else

Find an acceptable $x_{+}^{n}$ in the Newton direction $d_{n}$ using the line search Algorithm A6.3.1, page 325 [7]

Find an acceptable $x_{+}^{t}$ in the tensor direction $d_{t}$ using the line search Algorithm A6.3.1, page 325 [7]

if $f\left(x_{+}^{n}\right)<f\left(x_{+}^{t}\right)$ then

$$
x_{+}=x_{+}^{n}
$$

else

$$
x_{+}=x_{+}^{t}
$$

endif

endif

endif

5.2. else

Find an acceptable $x_{+}^{n}$ in the Newton direction $d_{n}$ using Algorithm A6.3.1, page 325 [7]

$$
x_{+}=x_{+}^{n}
$$

endif

6. $x_{c}=x_{+}, f\left(x_{c}\right)=f\left(x_{+}\right)$, go to step 1 
In step 1, the gradient is either computed analytically or approximated by the algorithm A5.6.3 given in Dennis and Schnabel [7]. In step 2, the Hessian matrix is either calculated analytically or approximated by a graph coloring algorithm described in [6]. In step 4.3, we first compute the tensor step $\delta$ of the transformed model (obtained by substituting $\hat{d}+\delta$ for $d$ in (1.3), where $\hat{d}$ is the global step computed in the previous iteration)

$$
\begin{aligned}
M_{T}\left(x_{c}+d\right)= & f\left(x_{c}\right)+\nabla f\left(x_{c}\right) \cdot \hat{d}+\frac{1}{2} \nabla^{2} f\left(x_{c}\right) \cdot \hat{d}^{2}+\frac{1}{2}\left(b^{T} \hat{d}\right)\left(s^{T} \hat{d}\right)^{2} \\
& +\frac{\gamma}{24}\left(s^{T} \hat{d}\right)^{4}+\left(\nabla f\left(x_{c}\right)+\nabla^{2} f\left(x_{c}\right) \hat{d}+\left(b^{T} \hat{d}\right)\left(s^{T} \hat{d}\right) s\right. \\
& \left.+\frac{1}{2}\left(s^{T} \hat{d}\right)^{2} b+\frac{\gamma}{24}\left(s^{T} \hat{d}\right)^{3} s\right) \cdot \delta+\frac{1}{2}\left(\nabla^{2} f\left(x_{c}\right)\right. \\
& +\left(b^{T} \hat{d}+\frac{\gamma}{2} s s^{T}\right) \cdot \delta^{2}+\left(s^{T} \hat{d}\right)\left(b^{T} \delta\right)\left(s^{T} \delta\right)+\frac{1}{2}\left(b^{T} \delta\right)\left(s^{T} \delta\right)^{2} \\
& +\frac{\gamma}{6}\left(s^{T} \hat{d}\right)\left(s^{T} \delta\right)^{3}+\frac{\gamma}{24}\left(s^{T} \delta\right)^{4} .
\end{aligned}
$$

Then we set the tensor step $d$ of the original tensor model (1.3) to $\hat{d}+\delta$. In step 4.4 , we obtain a perturbation $\mu$ such as $\nabla^{2} f\left(x_{c}\right)+\mu I$ is safely positive definite by using the Cill, Murray, Ponceleon, and Saunders method [9]. After we compute the $\mathrm{LDL}^{\mathrm{T}}$ of the Hessian matrix using the MA27 package [8], we change the block diagonal matrix $D$ to $D+E$. The modified matrix is block diagonal positive definite. This guarantees that the decomposition $L(D+E) L^{T}$ is positive definite as well. Note that the Hessian matrix is not modified if it is already positive definite. In step 5, we perform a standard backtracking line search global strategy to compute a next iterate $x_{+}$. The line search tensor method is much simpler to implement and to understand than the two-dimensional trust region tensor method introduced in [4], and is appreciably faster. For these reasons, this software uses a line search method. The global framework for the line search method we used in conjunction with our tensor method for large, sparse unconstrained optimization is similar to the one used for systems of nonlinear equations $[3,5]$. This strategy has proved very successful for large, sparse systems of nonlinear equations. This approach always tries the full tensor step first. If this provides enough decrease in the objective function, then we terminate; otherwise we find acceptable next iterates in both the Newton and tensor directions and select the one with the lower function value as the next iterate. The Newton step $d_{n}$ (if needed) is computed as a by-product of the minimization of the tensor model. It is the modified Newton step $\left(\nabla^{2} f\left(x_{c}\right)+\mu I\right)^{-1} \nabla f\left(x_{c}\right)$, where $\mu=0$ if $\nabla^{2} f\left(x_{c}\right)$ is safely positive definite, and $\mu>0$ otherwise. The stopping criteria of Algorithm 2.1 are described by the parameter TERMCD in 85 .

\section{Overview of the Software Package}

The required input to the software is the number of variables $\mathrm{N}$, the function $\mathrm{FCN}$ that computes $f(x)$, an initial guess $x_{0}$, the number of nonzeros $\mathrm{NZ}$ stored in the lower or upper half of the Hessian matrix, and the row and column indices of these nonzeros given in any order.

Two methods of calling the package are provided. In the short version, the user supplies only the above information, and default values of all other options are used. These include the calculation of the gradient and Hessian matrix by finite differences, and the use of the tensor 
rather than the standard Newton method. In the other method for calling the package, the user may override any default values of the package options.

The user has the option to choose between the tensor method and the standard Newton method. If the flag METHOD is set to 0, the package will use the standard method. The tensor method is used otherwise.

Upon completion, the program returns with an approximation XPLS to the minimizer $x_{*}$, the value of the objective function FPLS at XPLS, the value of the gradient GPLS (XPLS), the Hessian $H(X P L S)$, and a flag specifying under which stopping condition the algorithm has terminated.

The software package is coded so that if the user inputs the typical magnitude TYPX $i$ of each component of $x$, the performance of the package is the equivalent to what would result from redefining the independent variable $x$ with

$$
x_{\text {scaled }}=\left[\begin{array}{ccc}
1 / \mathrm{TYPX}_{1} & & \\
& \cdot \cdot \cdot & \\
& & \\
& & 1 / \mathrm{TYPX}_{n}
\end{array}\right] \cdot x
$$

and then running the package without scaling. The default value of each TYPX $X_{i}$ is 1 . Scaling is often important to use for problems in which the variable components are widely different in magnitudes.

The user may supply analytic routines for the gradient and/or the Hessian. If they are not supplied the package computes them by finite differences. The parameters GRDFLG and HSNFLG specify whether analytic gradient and Hessian have been provided, respectively. When the analytic gradient and/or Hessian are supplied, the user has the option of checking the supplied analytic routines against the package's finite difference routines.

The standard (default) output from this package consists of printing the input parameters and the final results. The printed input parameters are those used by the algorithm and hence include any corrections made by the program module OPTCHK, which examines the input specifications for illegal entries and consistency. The program will provide an error message if it terminates as a result of input errors. The printed results include a message indicating the reason for termination, an approximation XPLS to the solution $x_{*}$, the function value at XPLS, and the gradient vector GPLS. The package provides an additional means for the control of output via the variable MSG described in $\$ 5$. The standard output is the input state, the final results, and the stopping conditions. The user may suppress all output or may print the intermediate iteration results in addition to the standard output.

If the user sets the variable INFORM to 1 , then the package uses reverse communication to obtain the multiplication of the Hessian matrix at the current iterate by a given vector. If INFORM is set to 0 , then this quantity is computed by the subroutine MATMV provided by the package. 


\section{Interfaces and Usage}

Two interfaces have been provided with the package. If the user wishes to use all the defaults options provided by the package, then he should call TENSPDO (TENSPSO if single-precision is used). Only the required input described in $\$ 3$ needs to be supplied. The other interface, TENSPD (TENSPS if single-precision is used), requires the user to supply all parameters. The user may specify selected parameters only by first invoking the subroutine DFAULT, which sets all parameters to their default values, and then overriding only the desired values.

The two calling sequences are as follows.

1. CALL TENSPDO (NMAX, N, XO, NZ, IRN, LIRN, ICN, LICN, FCN, D1FN, D2FN,

* TYPX, MSG, XPLS, FPLS, GPLS, H, WRK, LWRK, IWRK, LIWRK, TERMCD)

2. CALL DFAULT(N, TYPX, FSCALE, GRADTL, STEPTL, ILIM, STEPMX,

* IPR, METHOD, GRDFLG, HSNFLG, NDIGIT, INFORM, MSG)

C USER OVERRIDES SPECIFIC DEFAULT VALUES PARAMETERS, E.G.

$\begin{array}{ll}\text { GRADTL } & =1.00-6 \\ \text { ILIM } & =500 \\ \text { GRDFLG } & =1 \\ \text { HSNFLG } & =1\end{array}$

CALL TENSPD (NMAX, N, XO, NZ, IRN, LIRN, ICN, LICN, FCN, GRD, HSN,

* TYPX, FSCALE, GRADTL, STEPTL, ILIM, STEPMX, IPR, METHOD,

* GRDFLG, HSNFLG, NDIGIT, MSG, XPLS, FPLS, GPLS, H, WRK, LWRK,

* IWRK, LIWRK, TERMCD, VECTOR, INFORM)

\section{Parameters and Défault Values}

The parameters used in the calling sequences of $\$ 4$ are fully described here. TENSPDO uses only those parameters that are preceded by an asterisk. When it is noted that module DFAULT returns a given value, this is the default employed by interface IENSPDO. The user may override the default value by utilizing TENSPD.

Following each variable name in the list below appears a one- or a two-headed arrow symbol of forms $\rightarrow,-$. and $\longrightarrow$. These symbols signify that the variable is for input, output, and input-output, respectively.

*NMAX $\rightarrow:$ A positive integer variable specifying the maximum dimension of the problem. This provision allows the user to solve several problems with different $N$ while using the same storage. Restriction: NMAX $\geq N$.

$* \mathrm{~N} \rightarrow$ : A positive integer variable specifying the number of variables in the problem. Restriction: $\mathbf{N} \geq 1$. 
*XO-: An array of length $\mathrm{N}$ that contains an initial estimate of the minimizer $x_{*}$.

$* \mathrm{NZ} \rightarrow$ : An integer variable that must be set by the user to the number of nonzeros stored in the lower or upper half of the Hessian matrix. It is not altered by the program. Restriction: $\mathrm{NZ} \geq 1$.

*IRN $\rightarrow:$ An integer array of length LIRN. On entry, it must hold the row index of each nonzero stored in the lower or upper half of the Hessian matrix.

*LIRN $\rightarrow:$ An integer variable that must be set by the user to the length of array IRN. LIRN need not be as large as LICN; normally it need not be very much greater than NZ. It is not altered by the program. Restriction: LIRN $\geq N Z$.

*ICN $\rightarrow$ : An integer array of length LICN. On entry, it must hold the column index of the nonzeros stored in lower or upper half of the Hessian matrix. On output, it holds the column indices of the factors of the Hessian.

$* \mathrm{LICN} \rightarrow:$ An integer variable that must be set by the user to the length of the Hessian array $\mathrm{H}$ and ICN. LICN should ordinarily be 2 to 4 times as large as NZ. It is not altered by the program. Restriction: LICN $\geq N Z$.

$* F C N \rightarrow$ : The name of a user supplied subroutine that evaluates the function $f$ at an arbitrary vector $x$. The subroutine must be declared EXTERNAL in the user's calling program and must conform to

$$
\text { CALL FCN }(X, F, N) \text {, }
$$

where $X$ is a vector of length $N$. The subroutine must not alter the values of $X$.

GRD $\rightarrow$ : The name of a user supplied subroutine that returns in $G$ the value of the gradient GRD must be declared EXTERNAL in the user's calling program and must conform to the usage

$$
\text { CALL GRD }(N, X, G) \text {, }
$$

where $X$ is a vector of length $N$, and $G$ is the gradient at $X$. GRD must not alter the values of $N$ and $X$. When using the interface TENSPD, if no analytic gradient is supplied (GRDFLG $=1$ ), the user must use the dummy name D1FN.

HSN $\rightarrow$ : The name of a user supplied subroutine that returns in $\mathrm{H}$ the value of the Hessian $\nabla^{2} f(x)$ at the current point X. HSN must be declared EXTERNAL in the user's calling program and must conform to the usage

$$
\operatorname{CALL} \operatorname{HSN}(N, X, H, N Z) \text {, }
$$

where $\mathbf{N}$ is the dimension of the problem, $X$ is the current point, $H$ is the Hessian at the current point, and $N Z$ is the number of nonzeros in $H$. HSN must not alter the values of $N R$, $N$, or $X$. 
Only the lower triangular part and the diagonal of $\mathrm{H}$ should be given. When using the interface TENSPD, if no analytic gradient is supplied (HSNFLG = 1), the user must use the dummy name D2FN.

*TYPX $\rightarrow:$ An array of length $\mathrm{N}$ in which the typical size of the components of $\mathrm{X}$ are specified. The typical component sizes should be positive real scalars. If a negative value is specified, its absolute value will be used. When 0 . is specified, 1 . will be used. The program will not abort. This vector is used by the the package to determine the scaling matrix $D_{x}$. Although the package may work reasonably well in a large number of instances without scaling, it may fail when the components of $x_{*}$ are of radically different magnitude and scaling is not invoked. If the sizes of the parameters are known to differ by many orders of magnitude, then the scale vector TYPX should definitely be used. Module DFAULT returns TYPX $=(1.0, \ldots, 1.0)$. For example, if it is anticipated that the range of values for the iterates $x_{k}$ is

$$
\begin{aligned}
& x_{1} \in\left[-10^{10}, 10^{10}\right] \\
& x_{2} \in\left[-10^{2}, 10^{4}\right] \\
& x_{3} \in\left[-6 \times 10^{-6}, 9 \times 10^{-6}\right]
\end{aligned}
$$

then an appropriate choice will be TYPX $=(1.0 \mathrm{E}+10,1.0 \mathrm{E}+3,7.0 \mathrm{E}-6)$.

FSCALE $\rightarrow$ : A positive real number estimating the magnitude of $f(x)$ near the minimizer $x_{*}$. It is used in the gradient stopping condition given below. If $f\left(x_{0}\right)$ is much greater than $f\left(x_{*}\right)$, FSCALE should be approximately $f\left(x_{*}\right)$. If a negative value is specified for FSCALE, its absolute value is used. When 0 . is specified, 1 . will be used. The program will not abort.

GRADTL $\rightarrow$ : Positive scalar giving the tolerance at which the scaled gradient of $f(x)$ is considered close enough to zero to terminate the algorithm. The scaled gradient is a measure of the relative change in $F$ in each direction $x_{i}$ divided by-the relative change in $x_{i}$. More precisely, the test used by the program is

$$
\max _{i}\left\{\frac{|\nabla f(x)|_{i} \max \left\{\left|x_{i}\right|, \text { TYPX }_{i}\right\}}{\max \{|f|, \text { FSCALE }\}}\right\} \leq \text { GRADTL. }
$$

The module DFAULT returns the value $\epsilon^{1 / 3}$. If the user specifies a negative value, the default value is used instead.

STEPTL-: A positive scalar providing the minimum allowable relative step length. STEPTL should be at least as small as $10^{-d}$, where $d$ is the number of accurate digits the user desires in the solution $x_{*}$. The actual test used is

$$
\max _{i}\left\{\frac{\left|x_{i}^{k}-x_{i}^{k-1}\right|}{\max \left\{\left|x_{i}{ }^{k}, \operatorname{TYPX}_{i}\right|\right\}}\right\} \leq \text { STEPTL }
$$

where $x^{k}$ and $x^{k-1}$ are the new and old iterates, respectively. The program may terminate prematurely if STEPTL is too large. Module DFAULLT returns the value $\epsilon^{2 / 3}$. If the user specifies a negative value, then the default value is used instead. 
ILIM $\rightarrow$ Positive integer specifying the maximum iterations to be performed before the program is terminated. Module DFAULT returns ILIM $=150$. If the user specifies ILIM $\leq 0$, the default value is used instead.

STEPMX $\rightarrow:$ A positive scalar providing the maximum allowable scaled step length $\left\|D_{x}\left(x_{+}-x_{c}\right)\right\|_{2}$, where $D_{x}=\operatorname{diag}\left(1 / \mathrm{TYPX}_{1}, \ldots, 1 / \mathrm{TYPX}_{n}\right)$. STEPMX is used to prevent steps that would cause the optimization problem to overflow, to prevent the algorithm from leaving the area of interest in parameter space, or to detect divergence in the algorithm. STEPMX should be chosen small enough to prevent these occurrences but should be larger than any anticipated "reasonable" step. The algorithm will halt and provide a diagnostic if it attempts to exceed STEPMX on five successive iterations. If a nonpositive value is specified for STEPMX, the default is used. Module DFAULT returns the value STEPMX $=\max \left\{\left\|x_{0}\right\|_{2} \cdot 10^{3}, 10^{3}\right\}$, where $x_{0}$ is the initial approximation provided by the user.

IPR $\rightarrow:$ The unit on which the routine outputs information. DFAULT returns the value 6 , which is the standard FORTRAN unit for the printer.

METHOD $\rightarrow:$ An integer flag designating which method to use.

METHOD $=0:$ Use Newton's method.

METHOD = 1: Use the tensor method.

Module DFAULT returns value 1. If the user specifies an illegal value, module OPTCHK will set METHOD to 1; the program will not abort.

GRDFLG $\rightarrow$ : Integer flag designating whether or not analytic Hessian has been supplied by the user.

GRDFLG $=0:$ No analytic gradient supplied.

GRDFLG $=1$ : Analytic gradient supplied (will be checked against finite difference gradient.)

GRDFLG $=2$ : Analytic gradient supplied (will not be checked against finite difference gradient.) When GRDFLG $=0$, the gradient is obtained by finite differences. The module DFAULT returns the value 0 . When GRDFLG $=1$ or 2 , the name of the user supplied routine that evaluates $\nabla f(x)$ must be supplied in GRD. When GRDFLG $=1$, the program compares the value of the user's analytic gradient routine at $x_{0}$ with a finite difference estimate and aborts if the relative difference between any two components is greater than 0.01. DFAULT returns GRDFLG $=0$. If the user specifies an illegal value, the module OPTCHK supplies the value 0 .

HSNFLG-: Integer flag designating whether or not analytic Hessian has been supplied by the 11ser.

HSNFLG $=0:$ No analytic Hessian supplied.

HSNFLG = 1: Analytic Hessian supplied (will be checked against finite difference Hessian.)

HSNFLG $=2$ : Analytic Hessian supplied (will not be checked against finite difference Hessian.) When HSNFLG $=0$, the Hessian is obtained by finite differences. The module DFAULT returns the value 0 . When HSNFLG $=0$, the Hessian values are computed by finite differences. When HSNFLG $=1$ or 2 , the name of the user-supplied routine that evaluates $\nabla^{2} f(x)$ must be sup- 
plied in HSN. When HSNFLG $=1$, the program compares the value of the user's analytic Hessian routine at $x_{0}$ with a finite difference estimate and aborts if the relative difference between any two components is greater than 0.01. DFAULT returns HSNFLG $=0$. If the user specifies an illegal value, the module OPTCHK supplies the value 0 .

NDIGIT $\rightarrow$ : Integer estimating the number of accurate digits on the objective function $f(x)$. DFAULT returns the value $-\mathrm{LOG}_{\mathbf{1 0}}(\epsilon)$, where $\epsilon$ is machine precision. If NDIGIT $\leq 0$ then the default value is used instead.

*MSG $\longleftrightarrow:$ An integer variable that the user may set on input to inhibit certain automatic checks or override certain default characteristics of the package. Currently, three "message" features can be used individually or in combination.

MSG $=0$ : No output will be produced.

$M S G=1:$ Print the input state, the final results, and the stopping conditions.

MSG $=2$ : Print the intermediate results, that is, the input state, each iteration including the current iterate $x_{k}, f(x)$, and $\nabla f(x)$, and the final results including the stopping conditions. The module DFAULT returns a value of 1 . On output, if the program has terminated because of erroneous input, MSG contains an error code indicating the reason:

MSG $=0:$ No error.

MSG $=-1$ : Mlegal dimension, $\mathbb{N} \leq 0$ or NMAX $<N$ was input. The program aborts.

MSG $=-2$ : Probable coding error in the user's analytic gradient routine GDR. Analytic and finite difference gradient do not agree within a tolerance of 0.01 . The program aborts. (This check can be overridden by setting GRDFLG $=2$.)

MSG $=-3$ : Probable coding error in the user's analytic Hessian routine HSN. Analytic and finite difference Hessian do not agree within a tolerance of 0.01 . The program aborts. (This check can be overridden by setting HSNFLG $=2$.)

*XPLS -: An array of length $\mathbf{N}$ containing the best approximation to the minimizer $x_{*}$ upon return. (If the algorithm has not converged, the last iterate is returned.)

*FPLS-: A scalar variable that contains the function value at the final iterate XPLS.

*GPLS-: An array of length $\mathrm{N}$ containing the gradient value at XPLS.

$\mathrm{H} \rightarrow$ : An array that is used to store the Hessian matrix at each iteration. It needs to be at least of dimension LICN. On exit, $H$ contains the Hessian value at the minimizer $x_{*}$.

*WRK $\rightarrow:$ An array of length LWRK. This is used as workspace by the package. Its length must be at least $8 *$ NMAX.

$*$ LWRK $\rightarrow:$ An integer variable. It must be set by the user to the length of array WRK and is not altered by the package.

*IWRK $\rightarrow$ : An integer array of length LIWRK. This is used as workspace by the package. Its 
length must be at least $10 * \operatorname{NMAX}+2$.

*LIWRK $\rightarrow:$ An integer variable. It must be set by the user to the length of array IWRK and is not altered by the package.

*TERMCD $\leftarrow:$ An integer that specifies the reason why the algorithm has terminated.

TERMCD $=1:$ The norm of the gradient at the final iterate was less than GRADTL.

TERMCD $=2:$ The length of the last step was less than STEPTL.

TERMCD $=3$ : Last global step failed to locate a point lower than XPLS. It is likely that either XPLS is an approximate solution of the function or STEPTL is too large.

TERMCD $=4:$ The iteration limit has been exceeded.

TERMCD $=5:$ Five consecutive steps of length STEPMX have been taken.

VECTOR $\leftrightarrow:$ An array of length N. It need not be set by the user on entry. If INFORM is set to 1 , a re-entry must be made with VECTOR set to H times VECTOR (see INFORM.)

INFORM $\longrightarrow:$ An integer variable. If it is set to 1 , the user must obtain $\mathrm{H}$ times VECTOR and re-enter TENSPD (TENSPS if single-precision is used) with INFORM unchanged. The result of $\mathrm{H}$ times VECTOR must be stored in VECTOR. The default value of INFORM is 0 , meaning that $H$ times VECTOR is computed by the package.

\section{Summary of Default Values}

The following parameters are returned by the module DFAULT:

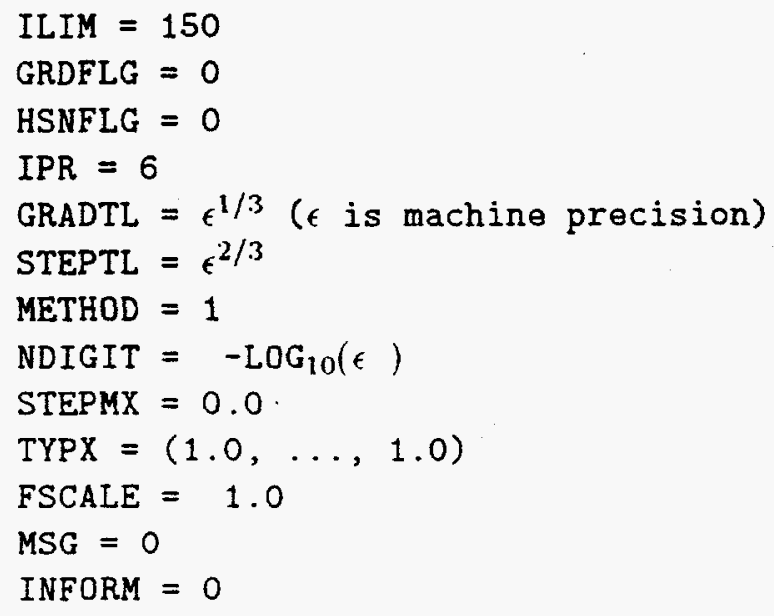

\section{Implementation Details}

This software package has been coded in Fortran 77 . The user has the choice between singleand double-precision versions. The user must then preprocess the package at compile time using either the tosngl or todble tools from CUTE [2], for the single- and double-precision 
versions, respectively. The tosngl program picks up the appropriate version by selecting any statement that begins with CS in the first column, where the $S$ character means that this is a single-precision version. On the other hand, the todble program picks up the appropriate version by selecting any statement that begins with $C D$ in the first column, with $D$ meaning that this is a double-precision version. Note that a statement that begins by neither CS nor CD will be picked by both tools.

The following software are included in the package:

1. Harwell MA27 package [8], which is used to compute the $\mathrm{L}^{\mathrm{T}} \mathrm{DL}$ factorization of the sparse Hessian matrix.

2. Gill-Murray-Ponceleon-Saunders code [9], which is used for modifying the negative eigencomponents of the Hessian matrix, in case this one is not safely positive definite.

3. The Coleman and Moreǵraph coloring algorithm [6], which is used for estimating a finitedifference approximation of a sparse Hessian matrix.

The program was developed and tested on a Sun SPARC 10 Model 40 computer.

The machine precision is calculated by the package and used in several places including finite differences stepsizes and stopping criteria. On some computers, the returned value may be incorrect because of compiler optimizations. The user may wish to check the computer value of the machine epsilon and, if it is incorrect, replace the code in the subroutine MCHEPS with the following statement

$$
\text { EPS = correct value of machine epsilon }
$$

\section{Example of Use}

In the example code shown in Figure 1, we first call the routine DFAULT, which returns the default values. We then override the values of ILIM, GRADTL and MSG. Next we call either the interface TENSPS or TENSPD for the single- and double-precision version, respectively, to solve the sparse unconstrained optimization problem coded in FCN. 


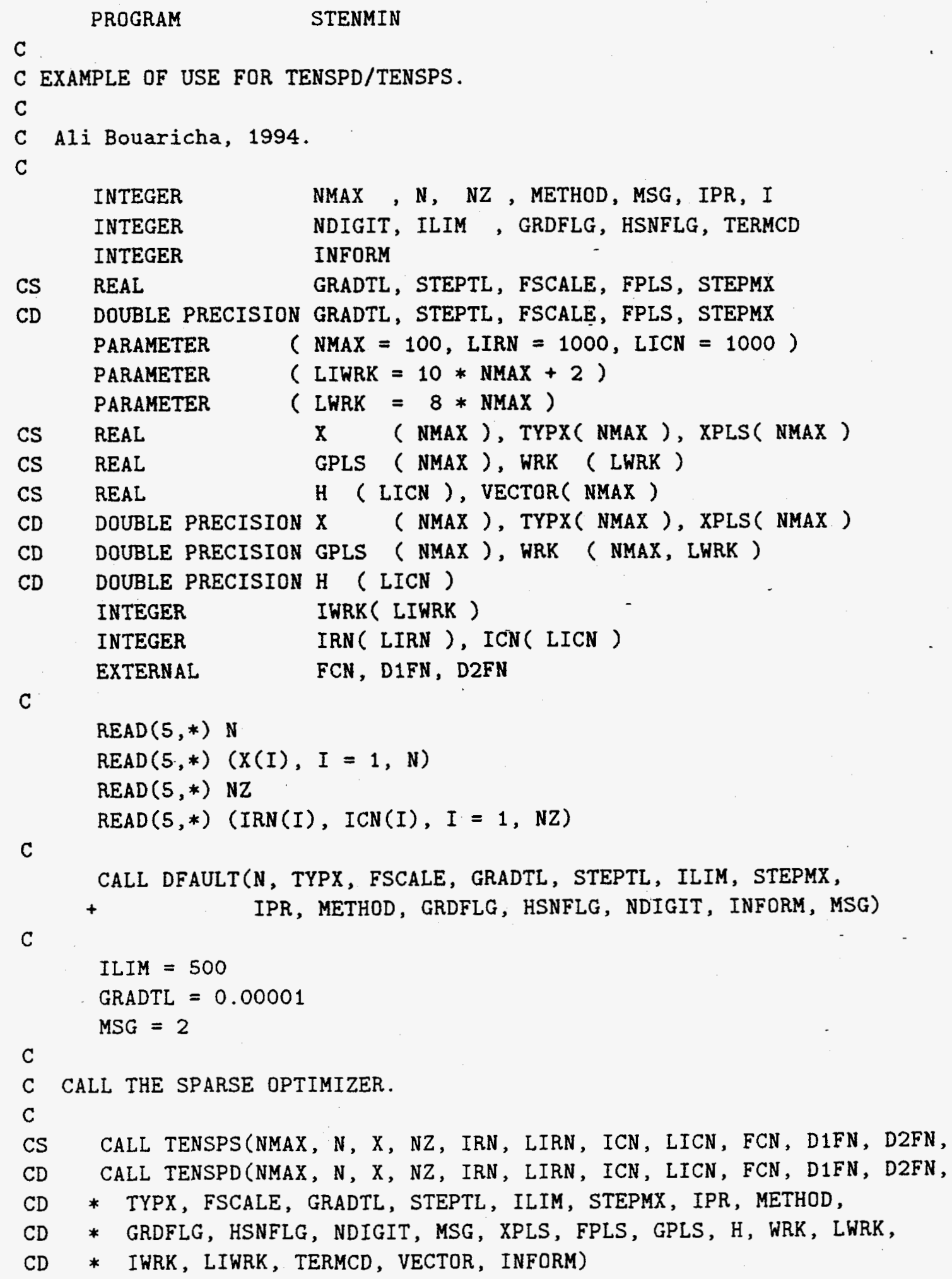




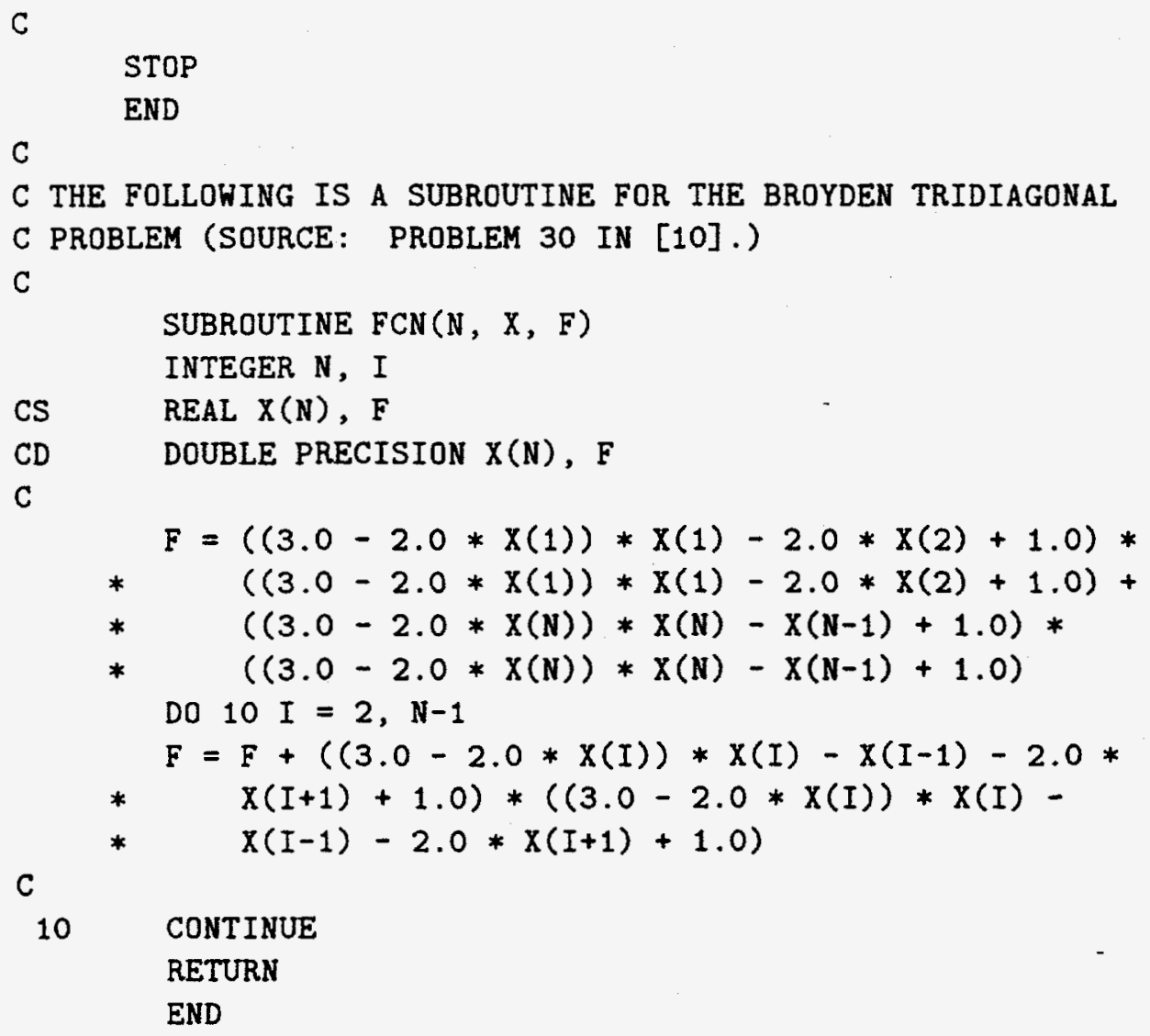

Figure 1. Code to solve a sparse unconstrained optimization problem

If we use the double-precision version of the package to solve the sparse unconstrained optimization problem given by $\mathrm{FCN}$, for the following input:

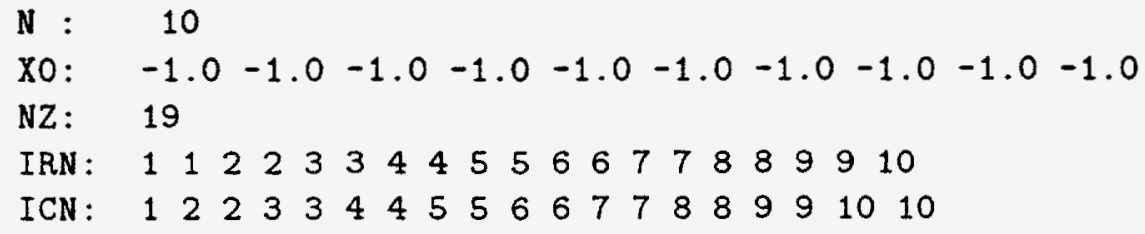

we obtain the following output:

$\begin{array}{llll}\text { OPTIM } & \text { TYPICAL X } & & \\ \text { OPTIM } & 0.1000000000000 D+01 & 0.1000000000000 D+01 & 0.1000000000000 D+01 \\ \text { OPTIM } & 0.1000000000000 D+01 & 0.1000000000000 D+01 & 0.1000000000000 D+01 \\ \text { OPTIM } & 0.1000000000000 D+01 & 0.1000000000000 D+01 & 0.1000000000000 D+01 \\ \text { OPTIM } & 0.1000000000000 D+01 & & \end{array}$


OPTIM

OPTIM

OPTIM

OPTIM

OPTIM

OPTIM

OPTIM

OPTIM

OPTIM

OPTIM

RESULT

RESULT

RESULT

RESULT

RESULT

RESULT

RESULT

RESULT

RESULT

RESULT

RESULT

RESULT

RESULT

OPTSTP

OPTSTP

RESULT

RESULT

RESULT

RESULT

RESULT

RESULT

RESULT

RESULT

RESULT

RESULT

RESULT

RESULT

RESULT
TYPICAL F

$0.1000000000000 D+01$

GRADIENT FLAG $=0$

HESSIAN FLAG $\quad=0$

METHOD $\quad=1$

ITERATION LIMIT $=500$

MACHINE EPSILON $=0.2220446049250 \mathrm{D}-15$

STEP TOLERANCE $=0.3666852862501 \mathrm{D}-10$

GRADIENT TOLERANCE $=0.1000000000000 \mathrm{D}-04$

MAXIMUM STEP SIZE $=0.3162277660168 \mathrm{D}+04$

ITERATION $\mathrm{K}=0$

$X(K)$

$-0.1000000000000 D+01$

$-0.1000000000000 D+01$

$-0.1000000000000 D+01$

$-0.1000000000000 D+01$

$-0.1000000000000 D+01$

$-0.1000000000000 D+01$

$-0.1000000000000 D+01$

$-0.1000000000000 D+01-0.1000000000000 D+01$

$-0.1000000000000 D+01$

FUNCTION AT $X(\mathrm{~K})$

$0.2100000000000 D+02$

GRADIENT AT $X(K)$

$-0.2599999804355 D+02$

$-0.3999998057019 D+01$

$-0.7999998136277 D+01$

$-0.7999998136277 \mathrm{D}+01$

$-0.7999998136277 \mathrm{D}+01$

$-0.7999998136277 \mathrm{D}+01$

$-0.7999998136277 D+01$

$-0.7999998136277 D+01$

$-0.3799999783194 D+02$

RELATIVE GRADIENT CLOSE TO ZERO.

CURRENT ITERATE IS PROBABLY SOLUTION.

ITERATION $K=9$

$X(K)$

$-0.5707221657357 D+00$

$-0.7055106888506 \mathrm{D}+00$

$-0.6818070022789 D+00$

$-0.7022101317047 D+00$

$-0.6918893109300 D+00$

$-0.7049061906923 D+00$

$-0.7014966362260 \mathrm{D}+00$

$-0.6657965030791 D+00$

$-0.5960350903456 \mathrm{D}+00$

$-0.4164122389914 D+00$

FUNCTION AT $\mathrm{X}(\mathrm{K})$

$0.1451030732465 \mathrm{D}-12$

GRADIENT AT $X(K)$

$0.4456254476679 D-06$

$0.2759511839662 D-07$

$0.4973660441711 \mathrm{D}-06$

$-0.5187295319932 D-06$

$0.1411968231618 \mathrm{D}-05$

$-0.40396710190790-06$

$0.2644468289644 \mathrm{D}-05$

$0.9521704223727 \mathrm{D}-06$

$0.1439646308990 \mathrm{D}-05$ 


\section{Test Results}

We tested our tensor and standard methods on the set of unconstrained optimization problems from the CUTE [2] and the MINPACK-2 [1] collections. Most of these problems have nonsingular Hessians at the solution. We also created singular test problems as proposed in [3, 12] by modifying the nonsingular test problems from the CUTE collection. The dimensions of these problems range from 100 to 10000 . All our computations were performed on a Sun SPARC 10 Model 40 machine using double-precision arithmetic.

A summary for the test problems whose Hessians at the solution have ranks $n, n-1$, and $n-2$ is presented in Table 1 . The descriptions of the test problems and the detailed results are given in [4]. In Table 1 the columns "better" and "worse" represent the number of times the tensor method was better and worse, respectively, than Newton's method by more than one gradient evaluation. The "tie" column represents the number of times the tensor and standard methods required within one gradient evaluation of each other. For each set of problems, we summarize the comparative costs of the tensor and standard methods using average ratios of three measures: gradient evaluations, function evaluations, and execution times. The average gradient evaluation ratio (geval) is the total number of gradients evaluations required by the tensor method. divided by the total number of gradients evaluations required by the standard method on these problems. The same measure is used for the average function evaluation (feval) and execution time (time) ratios. These average ratios include only problems that were successfully solved by both methods. We have excluded all cases where the tensor and standard methods converged to a different minimizer. However, the statistics for the "better," "worse," and "tie" columns include the cases where only one of the two methods converges, and exclude the cases where both methods do not converge. We also excluded problems requiring a number of gradient evaluations less or equal than 3 by both methods. Finally, columns " $t / s$ " and "s/t" show the number of problems solved by the tensor method but not by the standard method and the number of problems solved by the standard method but not by the tensor method, respectively.

The improvement by the tensor method over the standard method on problems with rank $n-1$ is dramatic, averaging $49 \%$ in function evaluations, $52 \%$ in gradient evaluations, and $60 \%$ in execution times. This is due in part to the rate of convergence of the tensor method being faster than that of Newton's method, which is known to be only linearly convergent with constant $\frac{2}{3}$. A typical convergence rate of the tensor method on rank $n-1$ problems is around 0.01 . Whether this is a superlinear convergence remains to be proved. On problems with rank $n-2$, the improvement by the tensor method over the standard method is also substantial, averaging $34 \%$ in function evaluations, $37 \%$ in gradient evaluations, and $38 \%$ in execution times. In the test results obtained for the nonsingular problems, the tensor method is only $2 \%$ better than the standard method in function evaluations, but $32 \%$ and $37 \%$ better in gradient evaluations and in execution times, respectively. The tensor method requires on the average more function evaluations than the standard method on some nonsingular problems. This is because the full tensor step does not provide sufficient decrease in the objective function, and therefore the tensor method has to perform a line search method in both the Newton and tensor directions, which causes the number of function evaluations required by the tensor method to be inflated.

The tensor method solved a total of four nonsingular problems, five rank $n-1$ problems, 
Table 1: Summary of the CUTE and MINPACK-2 test problems using line search

\begin{tabular}{|c|c|c|c|c|c|c|c|c|}
\hline Rank & \multicolumn{2}{|c|}{ Tensor/Standard } & \multicolumn{2}{c|}{ Pbs Solved } & \multicolumn{3}{c|}{ Average Ratio-Tensor/Standard } \\
\hline$\nabla^{2} f\left(x_{*}\right)$ & better & tie & worse & t/s & s/t & feval & geval & time \\
\hline$n$ & 54 & 38 & 4 & 4 & 0 & 0.98 & 0.68 & 0.63 \\
$n-1$ & 18 & 2 & 0 & 5 & 0 & 0.51 & 0.48 & 0.40 \\
$n-2$ & 18 & 1 & 1 & 7 & 0 & 0.66 & 0.63 & 0.62 \\
\hline
\end{tabular}

and 7 rank $n-2$ problems, stil Newton's method failed to solve. The reverse never occurred. This clearly indicates that the tensor method is most likely to be more robust than Newton's method.

The overall results show that the tensor method is more efficient than the standard method in solving large, sparse unconstrained optimization problems. Furthermore, the tensor method is likely to solve a wider range of problems.

Acknowledgments. I am grateful to Nick Gould for his assistance and encouragements. I also thank my CERFACS colleague Jacko Koster for reviewing this paper. 


\section{References}

[1] B. M. Averick, R. G. Carter, J. J. Moré, and G. L. Xue. The MINPACK-2 test problem collection. Technical Report ANL/MCS-P153-0692, Argonne National Laboratory, Argonne, USA, 1992.

[2] I. Bongartz, A. R. Conn, N. I. M. Gould, and Ph. L. Toint. CUTE: Constrained and Unconstrained Testing Environment. Technical Report TR/PA/93/10, Centre Européen de Recherche et de Formation Avancée en Calcul Scientifique (CERFACS), Toulouse, France, 1993.

[3] A. Bouaricha. Solving large sparse systems of nonlinear equations and nonlinear least squares problems using tensor methods on sequential and parallel computers. Ph.D. thesis, Computer Science Department, University of Colorado at Boulder, 1992.

[4] A. Bouaricha. Tensor methods for large, sparse unconstrained optimization. Technical Report TR/PA/94/02, Centre Européen de Recherche et de Formation Avancée en Calcul Scientifique (CERFACS), Toulouse, France, 1994.

[5] A. Bouaricha and R. B. Schnabel. TENSOLVE: a software package for solving systems of nonlinear equations and nonlinear least squares problems using tensor methods. Technical Report TR/PA/93/23, Centre Européen de Recherche et de Formation Avancée en Calcul - Scientifique (CERFACS), Toulouse, France, 1993.

[6] T. F. Coleman, B. S. Garbow, and J. J. Moré. Estimating sparse Hessian matrices. ACM Trans. Math. Software, 11:363-377, 1985.

[7] J. E. Dennis and R. B. Schnabel. Numerical methods for unconstrained optimization and nonlinear equations. Prentice-Hall, Englewood Cliffs, N.J., 1983.

[8] I. S. Duff and J. K. Reid. MA27: A set of Fortran subroutines for solving sparse symmetric sets of linear equations. Technical Report R-105.33, AERE Harwell Laboratory, Harwell, UK, $198: 3$.

[9] P. E. Gill, W. Murray, D. B. Ponceleon, and M. A. Saunders. Preconditioners for indefinite systems arising in optimization and nonlinear least squares problems. Technical Report SOL 90- . Department of Operations Research, Stanford University, California, 1990.

[10] J. J. Moré, B.S. Garbow, and K. E. Hillstrom. Testing unconstrained optimization software. AC.M Trans. Wath. Software, $7(1): 17-41,1981$.

[11] R. B. Schnabel and T. Chow. Tensor methods for unconstrained optimization using second derivatives. SIAM J. Optimization, 1:293-315, 1991.

[12] R. B. Schnabel and P. D. Frank. Tensor methods for nonlinear equations. SIAM J. Numer. Anal., $21: 815-843,1984$. 